POS $\quad$ PROCEEDINGS

\title{
Improving the LPM effect in BAMPS
}

\author{
Florian Senzel* \\ Institut für Theoretische Physik, Goethe-Universität Frankfurt, Max-von-Laue-Str. 1, D-60438 \\ Frankfurt am Main, Germany \\ E-mail: senzel@th.physik.uni-frankfurt.de

\section{Moritz Greif} \\ Institut für Theoretische Physik, Goethe-Universität Frankfurt, Max-von-Laue-Str. 1, D-60438 \\ Frankfurt am Main, Germany \\ E-mail: greifeth.physik.uni-frankfurt.de

\section{Carsten Greiner} \\ Institut für Theoretische Physik, Goethe-Universität Frankfurt, Max-von-Laue-Str. 1, D-60438 \\ Frankfurt am Main, Germany \\ E-mail: carsten.greinereth.physik.uni-frankfurt.de
}

Several analytic calculations of radiative jet energy loss demonstrated the importance of the non Abelian Landau-Pomeranchuk-Migdal (LPM) effect, a coherence effect resulting from the finite formation time of emitted gluons. While in these calculations it is possible to calculate gluon emissions including the LPM effect by rigorously resumming diagrams to any order in the opacity, it is still not straightforward to consider such coherence effects in dynamical transport simulations. Therefore we revisit in this proceeding the implementation of the LPM effect in the partonic transport approach BAMPS. By using Debye screened leading-order pQCD matrix elements for the elastic and an improved Gunion-Bertsch approximation for the inelastic processes, BAMPS simulates both the jet and medium evolution in ultra-relativistic heavy-ion collisions. We present a comparison between an effective modeling of the LPM effect via a theta function in the radiative matrix elements and a stochastic Ansatz for the suppression of gluon radiations. Finally, we discuss possible consequences of both methods for jet quenching observables.

International Conference on Hard and Electromagnetic Probes of High-Energy Nuclear Collisions 30 September - 5 October 2018

Aix-Les-Bains, Savoie, France

\footnotetext{
${ }^{*}$ Speaker.
} 


\section{Introduction}

When traversing the hot and dense matter produced in ultra-relativistic heavy-ion collisions [1], energetic partons lose energy by both elastic collisions and medium-induced gluon radiation. Several analytical calculations for the radiative energy loss $[2,3]$ demonstrated the dominance of the radiative energy loss for these jets. One important coherence effect for the radiative energy loss of partons is the non Abelian Landau-Pomeranchuk-Migdal (LPM) effect [4]: Due to the finite formation time of gluon emissions subsequent elastic interactions with the medium may act coherently to the gluon production. This results in a suppression of emissions in comparison to the BethlerHeitler regime where gluon emissions are independent from each other. While the LPM effect is calculable in analytic models by resumming arbitrary orders of diagrams, these calculations often suffer from kinematic approximations leading to e.g. the violation of energy-momentum conservation. One possible alternative is the application of numerical Monte Carlo generators. However, the connection between analytical formulations and their application in Monte Carlo simulations is not trivial. The first successful method to describe the LPM effect within a Monte Carlo approach was presented in Refs. [5, 6], where elastic scatterings of emitted gluons during the formation time modified the formation of the gluons. By additionally introducing a stochastic suppression factor, this approach is able to reproduce all parametric dependencies of the analytic BDMPS-Z formalism [7]. Other stochastic approaches for the LPM effect can be found in Refs. [8, 9].

In these proceedings we report on our status in improving the LPM effect in the partonic transport Boltzmann Approach to Multi-Parton Scatterings (BAMPS). To this end we discuss differences between two potential methods for modeling the LPM effect in a Monte Carlo model: first we revisit the previous modeling of the LPM effect via a parametric suppression in the radiative matrix element. After that we present our first attempt to model the LPM effect via a stochastic Ansatz. This method will show the expected non linear path-length dependence $\propto L^{2}$ of the radiative energy loss. However, as we will see, in contrast to other approaches of stochastic LPM formulations [5] our Ansatz does not describe the gluon emission spectrum $\frac{d N}{d \omega} \propto \omega^{-3 / 2}$ as expected in analytic formulations of the LPM effect. Finally, we present potential consequences of both methods for experimental observables.

\section{The LPM effect in BAMPS}

The partonic transport approach BAMPS [10, 11] describes the full 3+1D evolution of both the QGP medium as well as high energy particles traversing it by numerically solving the relativistic Boltzmann equation,

$$
p^{\mu} \partial_{\mu} f(\vec{x}, t)=\mathscr{C}_{22}+\mathscr{C}_{2 \leftrightarrow 3}
$$

for on-shell partons, quarks and gluons, and perturbative quantum chromodynamics (pQCD) interactions. To this end, a stochastical modeling of the collision probabilities together with a testparticle Ansatz is employed.

Within BAMPS, both elastic $2 \rightarrow 2$ scattering processes calculated in Debye-screened leadingorder pQCD, like e.g. $g g \rightarrow g g$, and inelastic $2 \leftrightarrow 3$ interactions, like e.g. $g g \leftrightarrow g g g$, based on 


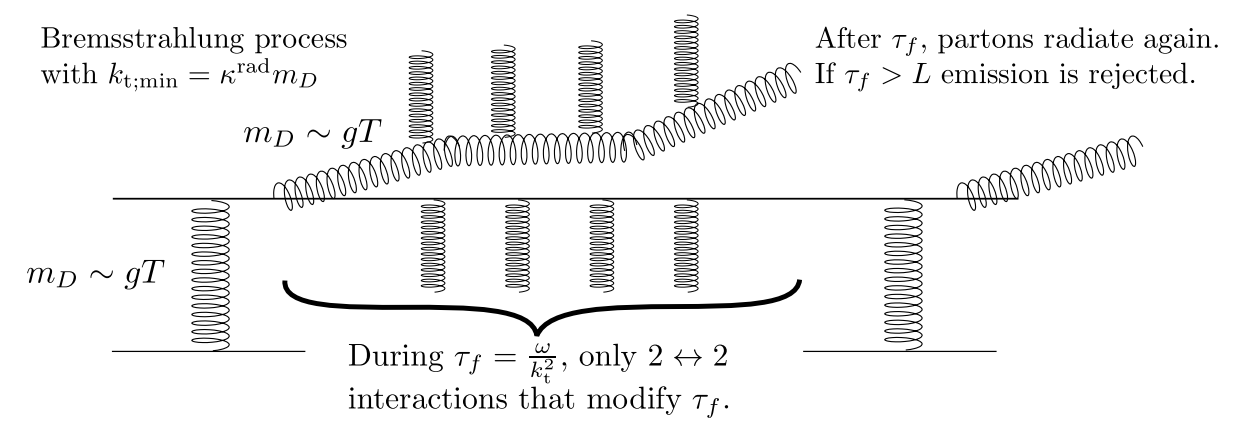

Figure 1: Scheme of the stochastic-LPM method in BAMPS.

an improved Gunion-Bertsch (GB) approximation $[12,13]$ are included. The matrix element for gluon emissions is

$$
\left|\overline{\mathscr{M}}_{X \rightarrow Y+g}\right|^{2}=48 \pi \alpha_{s}\left|\overline{\mathscr{M}}_{X \rightarrow Y}\right|^{2}(1-\bar{x})^{2}\left[\frac{\mathbf{k}_{\perp}}{k_{\perp}^{2}}+\frac{\mathbf{q}_{\perp}-\mathbf{k}_{\perp}}{\left(\mathbf{q}_{\perp}-\mathbf{k}_{\perp}\right)^{2}+m_{D}^{2}\left(\alpha_{S}\right)}\right]^{2}
$$

where $k_{\perp}$ and $q_{\perp}$ are the transverse momentum of the emitted and internal gluons, respectively. For more details about the general BAMPS framework and recent results we refer to Refs. $[10,11,14$, 15].

Previously, the LPM effect was modeled within BAMPS by an effective cutoff $\theta\left(\lambda-X_{\mathrm{LPM}} \tau_{f}\right)$ in the radiative matrix elements, where $\lambda$ is the mean free path of the radiating particle and $\tau_{f}=$ $\frac{\omega}{k_{\perp}^{2}}$ the gluon formation time. For $X_{\mathrm{LPM}}=1$, this procedure effectively allows only incoherent emissions, while $X_{\mathrm{LPM}}=0$ leads to the Bethe-Heitler regime of no LPM suppression. Therefore an implementation, where also some interference processes occur, should lead to a parameter in the region $0<X_{\mathrm{LPM}}<1$. For now, we treat $X_{\mathrm{LPM}}$ as a parameter and study its consequences for the radiative energy loss of partons in Sec. 3. As a remark, the infrared divergence of $k_{\perp}^{2}$ within the GB matrix element is effectively screened by the limit originating from the $\theta$ function. This parametric model of the LPM effect is called the " $\theta$-LPM method" throughout this paper.

The other method for modeling the LPM effect in this paper is a stochastic Ansatz similar to that of Ref. [5]: when an inelastic process calculated via the improved GB matrix element occurs, the emitted gluon is considered as not formed yet. During the formation time $\tau_{f}=\frac{\omega}{k_{\perp}^{2}}$ the gluon and its parent parton may only scatter elastically. These elastic scatterings modify the formation time allowing also collinear gluon emissions. After completing the formation time, both the parent parton and the emitted gluon may radiate again. All gluon emissions that are not completed within the medium length $L$ are rejected. This approach differs from other stochastic LPM approaches mainly in two points: (i) there is no suppression factor accounting for the difference between incoherent and coherent emissions, and (ii) during the formation time additional emissions are forbidden. Both differences simplify the numerical procedure in BAMPS significantly with the drawback that at the moment only incoherent gluon emissions can be reproduced. In contrast to the $\theta$-LPM method the infrared divergence of the GB matrix element is not cured by this method. Therefore we introduce a limit $k_{\perp \text {; min }}$ that should be of the order of the Debye mass, $k_{\perp} \propto m_{D}$, according to Ref. [16]. Therefore we introduce the parameter $\kappa^{\mathrm{rad}}$ defined by $k_{\perp ; \min }=\kappa^{\mathrm{rad}} m_{D}$ 
that controls the screening of the divergence. Fig. 1 summarizes the approach that we will call the "stochastic-LPM"(sLPM) method within this paper.

\section{Radiative energy loss in brick of QGP}
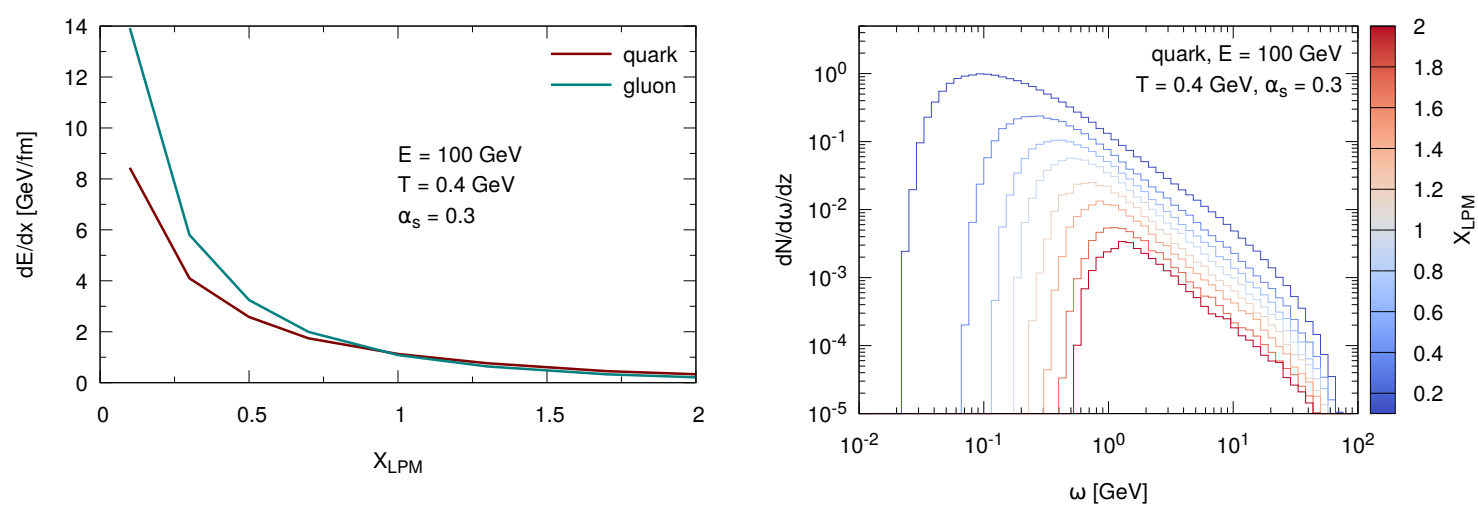

Figure 2: Sensitivity of the differential energy loss $\frac{\mathrm{d} E}{\mathrm{~d} x}$ (left) and gluon emission rate $\frac{\mathrm{d} N}{\mathrm{~d} \omega \mathrm{d} z}$ (right) on the parameter $X_{\mathrm{LPM}}$, for partons with $E=100 \mathrm{GeV}$ traversing a QGP with $T=0.4 \mathrm{GeV}$, in the $\theta$-LPM method.

As introduced in Sec. 2 the parameter $X_{\mathrm{LPM}}$ effectively controls the suppression within the $\theta$-LPM method. Fig. 2 shows the sensitivity on $X_{\mathrm{LPM}}$ for both the differential energy loss $\frac{\mathrm{d} E}{\mathrm{~d} x}$ (left) and the differential gluon emission rate $\frac{\mathrm{d} N}{\mathrm{~d} \omega \mathrm{d} z}$ (right) for a quark (gluon) with energy $E=100 \mathrm{GeV}$ that traverses a static brick of QGP with temperature $T=0.4 \mathrm{GeV}$ at a constant QCD coupling $\alpha_{s}=0.3$. The differential energy loss shows an approximately logarithmic dependence on the parameter $X_{\mathrm{LPM}}$. This dependence is caused by an increased rate of gluon emissions at small $X_{\mathrm{LPM}}$ due to the allowance of more collinear gluon emissions. In previous studies within BAMPS, we fixed the LPM parameter to $X_{\mathrm{LPM}}=0.3$ by a comparison to experimental data for the suppression of pions at RHIC [14].

Since the emission rate within the $\theta$-LPM method has no path-length dependence, the resulting radiative energy loss is only linearly depending on the path length. On the other hand, the gluon emissions within the stochastic-LPM have a path-length dependence due to the finite formation time. As shown in Fig. 3a this leads to a quadratic path-length dependence of the energy loss $\Delta E \propto L^{2}$ for small path-lengths $L \lesssim 1 \mathrm{fm}$. At larger medium lengths the energy loss again depends linearly $(\Delta E \propto L)$ on the path length. The reason for this different behavior is that gluons with larger formation time are suppressed for small medium path-lengths. This is in qualitative agreement with analytic calculations such as e.g. BDMPS-Z [7].

The resulting energy loss of the stochastic-LPM method is an interplay between the suppression due to the collinearity of the emission and the increased emission rate due to the soft divergence controlled by $\kappa^{\mathrm{rad}}$. While $\kappa^{\mathrm{rad}}=0.5$ shows the strongest energy loss, the value of $\kappa^{\mathrm{rad}}$ comes closer to the previously employed $\theta$-LPM with $X_{\mathrm{LPM}}=0.3$. 


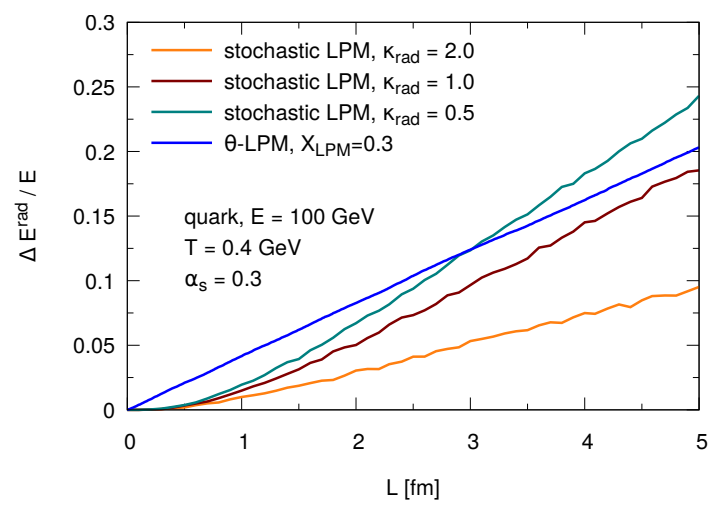

(a) Path-length dependence of radiative energy loss (b) Nuclear modification factor $R_{\mathrm{AA}}$ of LHC colliin a static medium.

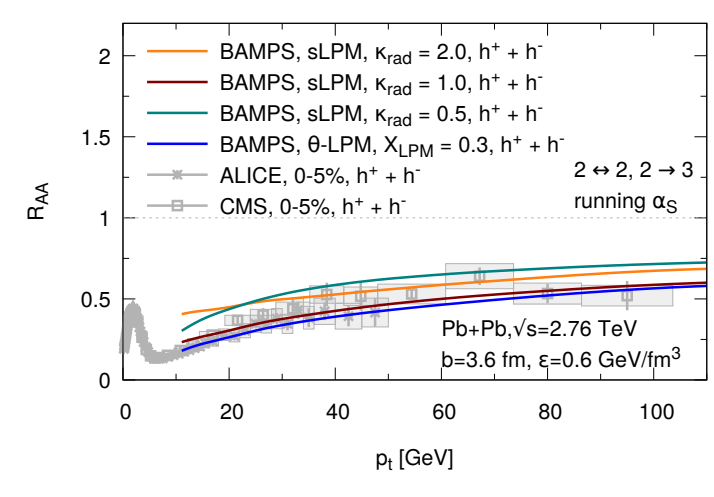

sions at $\sqrt{s}=2.76 \mathrm{TeV}$.

Figure 3: Comparison of the radiative energy loss of the $\theta$-LPM and stochastic-LPM method.

\section{Sensitivity of $R_{\mathrm{AA}}$ to LPM effect}

In order to study potential consequences of the stochastic-LPM method in BAMPS we present in Fig. $3 \mathrm{~b}$ the nuclear modification factor $R_{\mathrm{AA}}=\frac{\mathrm{d}^{2} N_{\mathrm{AA}} / \mathrm{d} p_{T} \mathrm{~d} y}{N_{\mathrm{bin}} \mathrm{d}^{2} N_{\mathrm{pp}} / \mathrm{d} p_{T} \mathrm{~d} y}$ calculated with the stochastic-LPM method together with the previous calculation within the $\theta$-LPM method with $X_{\mathrm{LPM}}=0.3 \mathrm{com}$ pared to experimental charged hadron data. As a remark, we employed for the calculation of $R_{\mathrm{AA}}$ a running coupling evaluated at the microscopic scale of each process. For more details we refer to Ref. [14]. Both LPM methods show a realistic description of the suppression of charged hadrons at $R_{\mathrm{AA}}$. Interestingly, the sensitivity to the parameter $\kappa^{\mathrm{rad}}$ is only modest in comparison to the previous parameter $X_{\mathrm{LPM}}$ of the $\theta$-LPM. The strongest suppression occurs for a value of $\kappa^{\mathrm{rad}}=1.0$.

\section{Conclusions}

We presented our first attempt to implement a stochastic method for calculating the non Abelian LPM effect within the partonic transport approach BAMPS. Due to the finite formation time of gluon emissions we find the expected quadratic path-length dependence as in other analytic formalisms. We compared this new approach to our previous modeling via a theta function. Both methods showed a realistic energy loss in terms of the nuclear modification factor $R_{\mathrm{AA}}$. Further studies will focus on the implementation of a stochastic suppression factor as in other models for the LPM effect [17]. These will allow us to also consider the difference between coherent and incoherent gluon emissions.

\section{Acknowledgments}

This work was supported by the Deutsche Forschungsgemeinschaft (DFG, German Research Foundation) - Project number 315477589 - TRR 211, HGS-HIRe, H-QM, and the Helmholtz International Center for FAIR within the framework of the LOEWE program launched by the State of Hesse. Numerical computations have been performed at the Center for Scientific Computing (CSC). 


\section{References}

[1] B. V. Jacak and B. Muller, Science (80-. ). 337 (2012) 310.

[2] A. Majumder and M. Van Leeuwen, Prog. Part. Nucl. Phys. 66, 41 (2011).

[3] N. N. Armesto, B. Cole, C. Gale, W. a. Horowitz, P. Jacobs, S. Jeon et al., Phys. Rev. C 86 (2012) 064904.

[4] A. B. Migdal, Phys. Rev. 103 (1956) 1811.

[5] K. Zapp, J. Stachel and U. A. Wiedemann, Phys. Rev. Lett. 103 (2009) 152302.

[6] K. C. Zapp, J. Stachel and U. A. Wiedemann, J. High Energy Phys. 2011 (2011) 118.

[7] R. Baier, D. Schiff and B. G. Zakharov, Annu. Rev. Nucl. Part. Sci. 50 (2000) 37.

[8] C. E. Coleman-Smith, S. A. Bass and D. Srivastava, Nucl. Phys. A 862-863 (2011) 275.

[9] W. Ke, Y. Xu and S. A. Bass, arXiv:1810.08177.

[10] Z. Xu and C. Greiner, Phys. Rev. C 71 (2005) 064901.

[11] Z. Xu and C. Greiner, Phys. Rev. C 76 (2007) 024911.

[12] J. F. Gunion and G. Bertsch, Phys. Rev. D 25 (1982) 746.

[13] O. Fochler, J. Uphoff, Z. Xu and C. Greiner, Phys. Rev. D 88 (2013) 014018.

[14] J. Uphoff, F. Senzel, O. Fochler, C. Wesp, Z. Xu and C. Greiner, Phys. Rev. Lett. 114 (2015) 112301.

[15] J. Uphoff, O. Fochler, Z. Xu and C. Greiner, J. Phys. G Nucl. Part. Phys. 42 (2014) 1.

[16] P. Arnold, G. D. Moore and L. G. Yaffe, J. High Energy Phys. 2002 (2002) 030.

[17] F. Senzel et al., in preparation, 2019. 